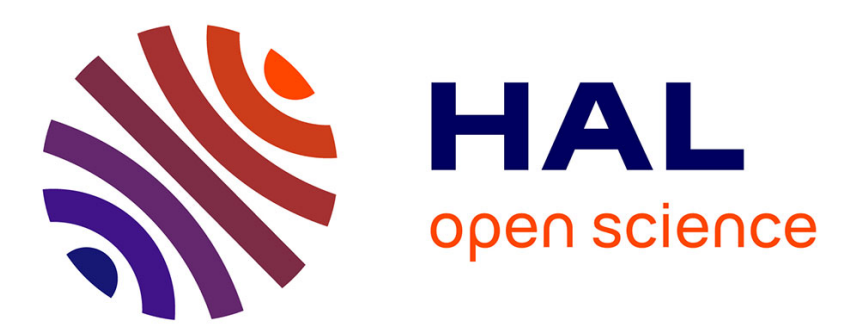

\title{
Diameter dependence of the optoelectronic properties of single walled carbon nanotubes determined by ellipsometry
}

\author{
Yann Battie, L. Broch, A. En Naciri, J.-S Lauret, Maud Guézo, A. Loiseau
}

\section{> To cite this version:}

Yann Battie, L. Broch, A. En Naciri, J.-S Lauret, Maud Guézo, et al.. Diameter dependence of the optoelectronic properties of single walled carbon nanotubes determined by ellipsometry. Carbon, 2015, 83, pp.32-39. 10.1016/j.carbon.2014.11.021 . hal-01230304

HAL Id: hal-01230304 https://hal-univ-rennes1.archives-ouvertes.fr/hal-01230304

Submitted on 18 Nov 2015

HAL is a multi-disciplinary open access archive for the deposit and dissemination of scientific research documents, whether they are published or not. The documents may come from teaching and research institutions in France or abroad, or from public or private research centers.
L'archive ouverte pluridisciplinaire HAL, est destinée au dépôt et à la diffusion de documents scientifiques de niveau recherche, publiés ou non, émanant des établissements d'enseignement et de recherche français ou étrangers, des laboratoires publics ou privés. 


\section{Diameter dependence of the optoelectronic properties of single walled carbon nanotubes}

\section{determined by ellipsometry.}

Y. Battie, ${ }^{1, *}$ L. Broch, ${ }^{1}$ A. En Naciri, ${ }^{1}$ J.-S. Lauret, ${ }^{2}$ M. Gicquel-Guézot ${ }^{3}$ A. Loiseau ${ }^{4}$

${ }^{1}$ LCP-A2MC, Université de Lorraine, 1 Bd Arago, 57070 Metz (France)

${ }^{2}$ Laboratoire Aimé Cotton, CNRS UPR 3321, ENS Cachan, 94245 Cachan, France

${ }^{3}$ FOTON, UMR 6082, INSA, Avenue des Buttes de Coësmes, 35043 Rennes, France

${ }^{4}$ Laboratoire d'étude des microstructures, ONERA-CNRS UMR 104, 29 Av. de la Division Leclerc, 92322 Chatillon, France

*Electronic address: yann.battie@univ-lorraine.fr.

We report ellipsometric measurement on single walled carbon nanotube (SWCNT) films performed in a large spectral range from $0.07 \mathrm{eV}$ to $4.97 \mathrm{eV}$. The complex dielectric functions of SWCNTs are correlated to their diameter distribution extracted from transmission electron microscopy. Here we show that the transition energies between Van Hove singularities are directly related to the strong one dimensional confinement. In the infrared spectral range, the real part of the dielectric function becomes negative. The electronic properties of SWCNTs are extracted from ellipsometry by using a Drude model. The mobility and the mean free path of charge carriers are limited by the high number of SWCNT contacts. In accordance with tight binding simulation, the conductivity and the charge carrier concentration increase with the SWCNT diameter. Finally, we demonstrate that the $\pi$-plasmon energy depends on the charge carrier concentration.

*Corresponding author: Fax: +33 0387315885

Electronic address: yann.battie@univ-lorraine.fr. 


\section{Introduction}

Single walled carbon nanotube (SWCNT) is a one dimensional material which has remarkable optical and electronic properties. Indeed, depending on its chirality, a SWCNT can be metallic or semiconductor. Moreover, semiconducting SWCNTs (SC-SWCNTs) have direct-bandgap, so they can be used to emit and detect light. These properties make SWCNT an ideal candidate for new optoelectronic devices such as transparent conductive electrodes[1], highbit-rate all-optical signal regenerator[2], high-repetition mode-locked laser[3] or bolometer[4]. However, the development and the optimization of such devices suffer to the lack of experimental reports on the optoelectronic properties of SWCNT film which are currently extracted from transport measurements. However, this technique requires electrical contacts and known current paths, which exclude the estimation of the intrinsic electronic properties of SWCNT assembled into percolated film in which Schottky barrier at the contacts are unambiguously observed[5-6].

Ellipsometry is a non contact characterisation tool which allows determining the complex dielectric function of material. The dielectric function contains several insights on the optoelectronic properties of materials such as the optical band gap or the electronic conductivity. Thus, the dielectric function is crucial to design optoelectronic devices. Simulations based on ab-initio calculation[7] or tight binding approximation[8] show a strong dependence between the dielectric function and the chirality of SWCNTs. Ellipsometry was previously used to determine the chirality distribution and the porosity of SWCNT films[9]. Using an effective medium approximation to analyse ellipsometric data, Fanchini et al.[10] claimed that for high SWCNT density, SWCNT film exhibits a metallic behaviour suggesting that the percolation threshold can be estimated by analysing the Drude contribution to the SWCNT dielectric function. In the particular case of oriented SWCNTs, ellipsometry have 
revealed that the optical anisotropy is related to anisotropic selection rule conditions[11]. The optoelectronic properties of boron doped SWCNT was investigated by combining ellipsometric and transport measurements[12]. In this reference, the authors claimed that boron functionalization shifts the Fermi level and induces an acceptor states in the density of state (DOS) of SWCNT. On the other hand, ellipsometric measurements performed on sorted SWCNTs have shown that the energy of the $\pi$-plasmon band depends on the ratio between SC-SWCNTs and metallic SWCNTs (M-SWCNTs)[13]. Despite several advances that have been made to measure the dielectric function of SWCNT, the correlation between the SWCNT diameter and the dielectric function is still under debate.

This paper focuses on the ellipsometric measurements of the complex dielectric function of SWCNT films in a large spectral range from $0.07 \mathrm{eV}$ to $4.97 \mathrm{eV}$. The influence of SWCNT diameter on the optical transitions and $\pi$-plasmon band is highlighted. Due to one dimensional confinement, we report a large dependence between the optical transition and the diameter. By adjusting the infrared part of dielectric function by a Drude model, the electronic DC conductivity of SWCNT film, charge carrier concentration, mean free path and mobility are estimated. The variation of charge carrier concentration is compared to the simulated one from tight binding calculation. We demonstrate that the mean free path of charge carrier is limited by the large number of contacts between SWCNTs. Finally, we highlight a direct correlation between charge carrier density and the $\pi$-plasmon energy of SWCNTs.

\section{Experimental}

\subsection{Dispersion of SWCNTs:}

Electrical arc discharge (EAD), high-pressure carbon monoxide (HiPCo), raw cobalt molybdenum catalyser (CoMoCat) SWCNTs and SWCNTs enriched in $(6,5)$ chirality, 
purchased from Carbon Solution inc., Unidym and SouthWest Nanotechnologies, respectively, are investigated. $4 \mathrm{mg} / \mathrm{ml}$ of SWCNT aqueous suspension was prepared by mixing in water, SWCNTs with $2 \%$ wt of sodium cholate. The suspension was sonicated during 90 min with an ultrasonic tip. The temperature of the suspension was fixed at $5^{\circ} \mathrm{C}$ with a thermostatic bath. The suspension was ultra-centrifuged at $35000 \mathrm{rpm}$ during $1 \mathrm{~h}$ to separate non-dispersed carbon nanotubes from the well separated ones. The supernatant was then extracted.

\section{$2.2(6,5)$ SWCNT chirality enrichment:}

SWCNTs with a nearly single $(6,5)$ chirality are sorted by density gradient ultracentrifugation from CoMoCat SWCNTs [14-15]. Briefly a non-linear density gradient was made by juxtaposing 9 layers in an ultracentrifugation column. The layers consist in $0 \%, 15 \%, 17.5 \%$, $20 \% 22.5 \%, 25 \%, 27.5 \%, 30 \%$ and $60 \%$ of iodixanol dissolved in water. These layers also contain $0.8 \%$ wt of sodium cholate. Then, a solution of CoMoCat SWCNTs with $25 \%$ of iodixanol and $0.8 \%$ wt of sodium cholate was introduced in the nonlinear density gradient. The gradient was then centrifuged during $14 \mathrm{~h}$ at $35000 \mathrm{rpm}$. The SWCNT micelles must move down through the density gradient column during the ultracentrifugation in order to reach their isopycnic points. A pink layer enriched in $(6,5)$ SWCNT was observed at the top of the gradient. This layer was extracted with the gradient master device (Biocomp).

\subsection{SWCNT film preparation and characterization}

SWCNTs are deposited on nitrocellulose filter with $0.22 \mu \mathrm{m}$ pore size by filtering the SWCNT suspension with a Buchner filtration set up. The volume of the SWCNT suspension was adjusted to obtain a completely black and opaque thick film. The film was then washed 
thoroughly to remove the surfactant. Note that contrary to reference [11], the SWCNT films are not transferred on a silicon substrate and nitrocellulose filter is used as substrate.

Electrical measurements are recorded at room temperature with an ECOPIA HMS-5500 set up. Briefly, four point probe measurements are performed in the Van der Paw geometry. The film resistivity is obtained by applying a current of $0.1 \mathrm{~mA}$ which flows along one edge of the sample and by measuring the voltage across the opposite edge. This set up can also be used to perform Hall measurements. A $0.51 \mathrm{~T}$ magnetic field is created perpendicularly to the film surface. A current is applied along two opposite edges of the sample and a voltage induced by the magnetic field is recorded between the other edges. This allows estimating the Hall coefficient of the film. The carrier density and mobility are deduced from the film resistivity and Hall coefficient. Note that the SWCNTs are assumed to be p-doped by air.

Transmission electron microscopy (TEM) investigations were performed with a Technai CM20 electron microscope operating at $200 \mathrm{kV}$. The TEM grids were prepared by depositing a drop of SWCNT suspension on a copper grid. Scanning electron microscopy (SEM) was performed with a field emission gun scanning electron microscope Zeiss Gemini operating at $15 \mathrm{kV}$. The optical properties of SWCNT film are investigated by spectroscopic ellipsometry. A home built rotating polariser ellipsometer and a photoelastic ellipsometer (Horiba Jobin Yvon) were used to cover a large spectral range from $0.07 \mathrm{eV}$ to $4.97 \mathrm{eV}$. The ellipsometric measurements were performed at three angles of incidence $\left(50^{\circ}, 60^{\circ}, 70^{\circ}\right)$.

\section{Results and discussion}

\subsection{SWCNT diameter distribution}

Figure 1 depicts the diameter distributions of SWCNT extracted from a statistical analysis based on transmission electron microscopy (TEM) of more than 50 SWCNTs. Due to Van 
Der Waals interactions, SWCNTs are assembled into bundles. The SWCNT diameters follow

a Gaussian distribution. The mean radius of $(6,5)$, CoMoCat, HiPCo and EAD SWCNTs are $0.75 \mathrm{~nm} \pm 0.08 \mathrm{~nm}, 0.75 \mathrm{~nm} \pm 0.08 \mathrm{~nm}, 0.95 \mathrm{~nm} \pm 0.09 \mathrm{~nm}$ and $1.3 \mathrm{~nm} \pm 0.12 \mathrm{~nm}$, respectively (Fig.1). Due to the limited TEM resolution few diameter differences are observed between $(6,5)$ and CoMoCat SWCNTs.
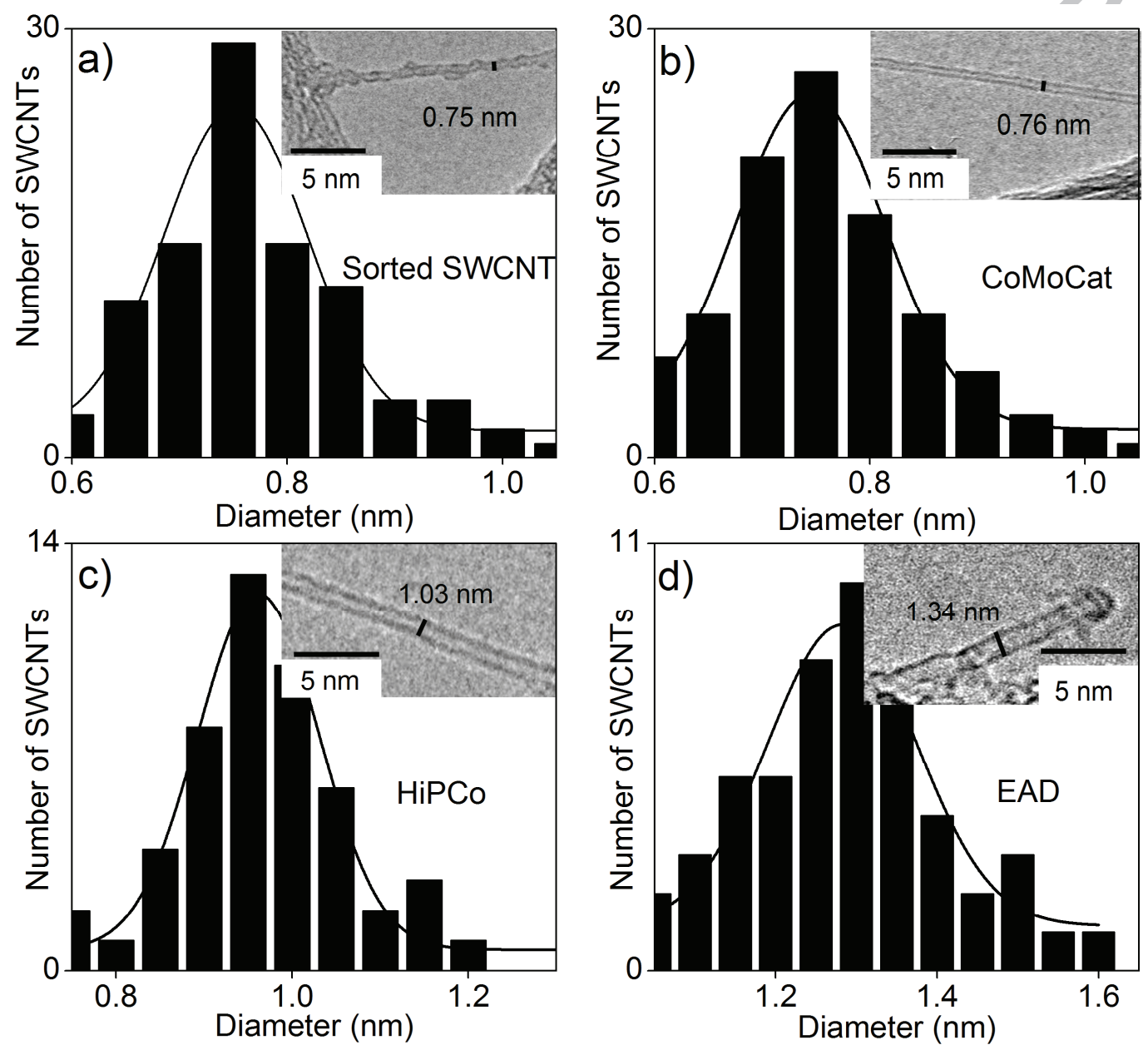

Fig. 1- Diameter distribution of (a) (6,5) enriched, (b) CoMoCat, (c) HiPCo and (d) EAD SWCNTs extracted from TEM images (in inset). 


\subsection{Ellipsometric data analysis}

Ellipsometric measurements were performed in the $0.07 \mathrm{eV}$ to $4.97 \mathrm{eV}$ spectral range on dense SWCNT films. Depending on the SWCNT film, the film thickness, estimated from mechanical profilometry, is in the $500 \mathrm{~nm}-800 \mathrm{~nm}$ range. These films are sufficiently thick, absorbent and reflective to be considered as an isotropic semi-infinite medium for the ellipsometric data analysis. In this context, the effective dielectric constant $\varepsilon$ of SWCNT films can be analytically derived from the measured ellipsometric angles $\Psi$ and $\Delta$ not shown[16]:

$$
\varepsilon=\sin ^{2} \theta\left(1+\left(\frac{1-\tan \Psi e^{j \Delta}}{1+\tan \Psi e^{j \Delta}} \tan \theta\right)^{2}\right)
$$

where $\theta$ is the angle of incidence. In other words, the effective dielectric function of SWCNTs (Fig.2) can be extracted from ellipsometric measurements by using equation (1) without fitting. Measurements performed at three angles of incidence $\left(50^{\circ}, 60^{\circ}, 70^{\circ}\right)$ reveal that the dielectric function does not depends on the angle of incidence (not shown). This unambiguously suggests that the surface roughness is sufficiently small to be neglected, confirming the correctness of our ellipsometric model. 
(a)

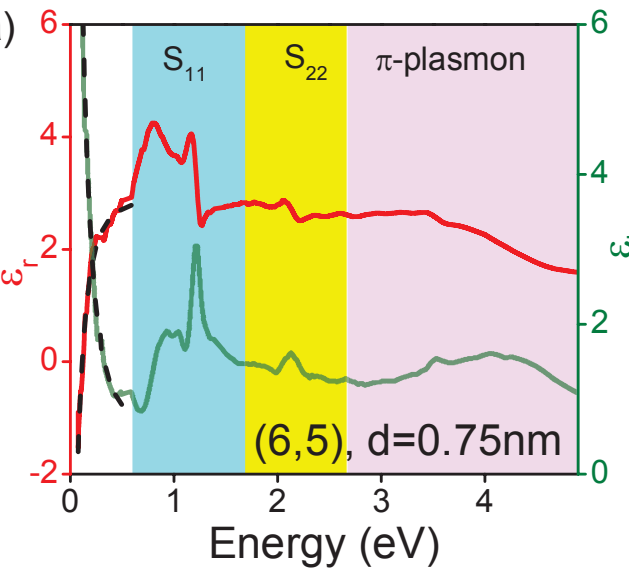

(c)

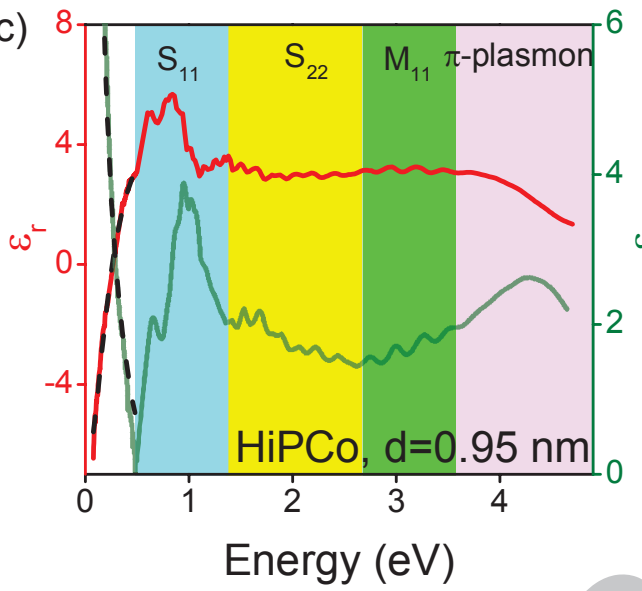

(b)

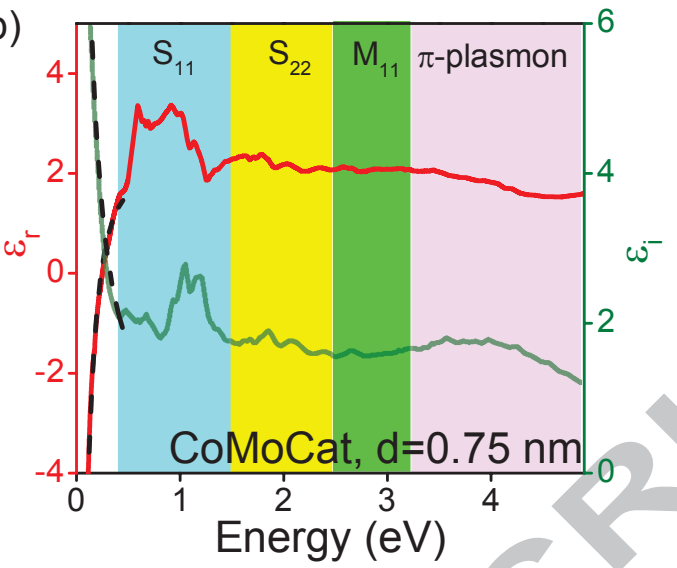

(d)

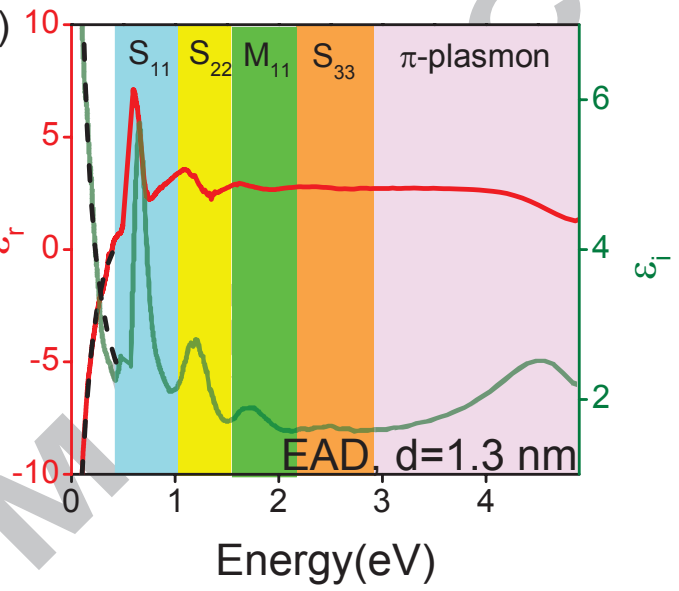

Fig. 2- Real (red line) and imaginary (green line) parts of dielectric function of (a) $(6,5)$ enriched, (b) CoMoCat, (c) HiPCo and (d) EAD SWCNTs. The Drude adjustment in the infrared spectral range is represented in black dash lines.

\subsection{Visible-near infrared optical properties of SWCNTs}

Several bands are observed from $0.40 \mathrm{eV}$ to $3.20 \mathrm{eV}$ in the $\varepsilon_{\mathrm{i}}$ spectra. These bands are associated to the transitions between the Van Hove singularities (VHS) in the SWCNT DOS induced by the one-dimensional nature of SWCNT. These bands are assigned to specific transitions between VHS according to references [17-22]. Indeed, the $\mathrm{S}_{11}, \mathrm{~S}_{22}, \mathrm{M}_{11}, \mathrm{~S}_{33}$ bands of EAD SWCNTs are in the 0.5-1eV[18], 1-1.5eV[19], 1.5-2.1eV[19] and 2.1-2.9eV[19] spectral ranges, respectively. The $\mathrm{S}_{11}, \mathrm{~S}_{22}, \mathrm{M}_{11}$, bands of HiPCo SWCNTs are in the 0.6$1.4 \mathrm{eV}[18], 1.4-2.6 \mathrm{eV}[19]$ and $2.6-3.5 \mathrm{eV}$ spectral ranges, respectively. The $\mathrm{S}_{11}, \mathrm{~S}_{22}, \mathrm{M}_{11}$, 
bands of CoMoCat SWCNTs are located in the 0.6-1.5eV[20], $1.5-2.5 \mathrm{eV}[20]$ and $2.6-3.1 \mathrm{eV}$ [20] spectral ranges, respectively. The transition energies associated to $(6,5)$ SWCNTs are given by Bachilo [17]. According to Knupfer et al. [21] and Lauret et al. [22], the band located above $3.5 \mathrm{eV}$ is the $\pi$-plasmon band of SWCNTs.

In agreement with the Kramers-Kronig relation, large variations of $\varepsilon_{\mathrm{r}}$ appear close to these transitions. The CoMoCat and HiPco SWCNT spectra are more structured than the EAD one. These substructures are the optical signature of individual chiralities present in the film. Due to their large diameter, EAD SWCNT has broad chirality distribution. This induces an inhomogeneous broadening of the absorption bands.

Transitions between the first $\left(\mathrm{S}_{11}\right)$ and second $\left(\mathrm{S}_{22}\right)$ pairs of VHS of SC-SWCNTs appear in all SWCNT spectra while the transition between their third VHS $\left(\mathrm{S}_{33}\right)$ is only observed in the EAD spectra. In the other hand, the amplitude of the band associated to the transitions between the first pair $\left(\mathrm{M}_{11}\right)$ of VHS of M-SWCNTs is reduced as the SWCNT mean diameter decreases and completely disappears for sorted SWCNTs. Indeed, CoMoCat SWCNTs contain few metallic species[9]. Moreover, the dielectric function of sorted SWCNTs is dominated by two wide bands located at $1.23 \mathrm{eV}$ and $2.15 \mathrm{eV}$ attributed to the $\mathrm{S}_{11}$ and $\mathrm{S}_{22}$ transitions of $(6,5)$ SC-SWCNTs[17], respectively, confirming the high $(6,5)$ chirality enrichment. As shown in Figure 2, the transition energies depend on the SWCNT source. As example, the $\mathrm{S}_{11}$ transition is centered at $1.23 \mathrm{eV}, 1.11 \mathrm{eV}, 0.99 \mathrm{eV}$ and $0.67 \mathrm{eV}$ for $(6,5)$ enriched, CoMoCat, HiPco, and arc discharge SWCNTs. By neglecting excitonic effects, these energies can be considered as the optical band gap of SC-SWCNTs[23]. The measured transitions energies are reported in a Kataura plot (Fig.3) and are compared to the calculated ones from tight binding approximation[24]. Experimental data are in close agreement with the calculated ones. Small deviations with theoretical value can be attributed to the perturbations 
by the environment and excitonic effects. In agreement with tight binding and zone-folding calculations[25], the measured energies $\mathrm{ES}_{\mathrm{ii}}$ and $\mathrm{EM}_{11}$ are inversely proportional to the SWCNT diameter $\mathrm{d}$ :

$$
E_{S_{i i}}=\frac{2 i a_{c c} \gamma_{0}}{d}, \text { (2) } E_{M_{11}}=\frac{6 a_{c c} \gamma_{0}}{d},(3)
$$

where ac-c and $\gamma_{0}$ are the carbon bond length and the transfer integral parameter used to describe the optoelectronic properties of SWCNTs, respectively. Thus, by fitting simultaneously all experimental transition energies by equations (2-3), the transfer integral can be estimated to $2.8 \mathrm{eV} \pm 0.1 \mathrm{eV}$. This value is in close agreement with the reported one by Dresselhaus et al.[25]. Thus, the redshift of the transition energies is due to the strong one dimensional confinement induced by the periodic boundary conditions that result from wrapping the graphene sheet into a tubular structure. 


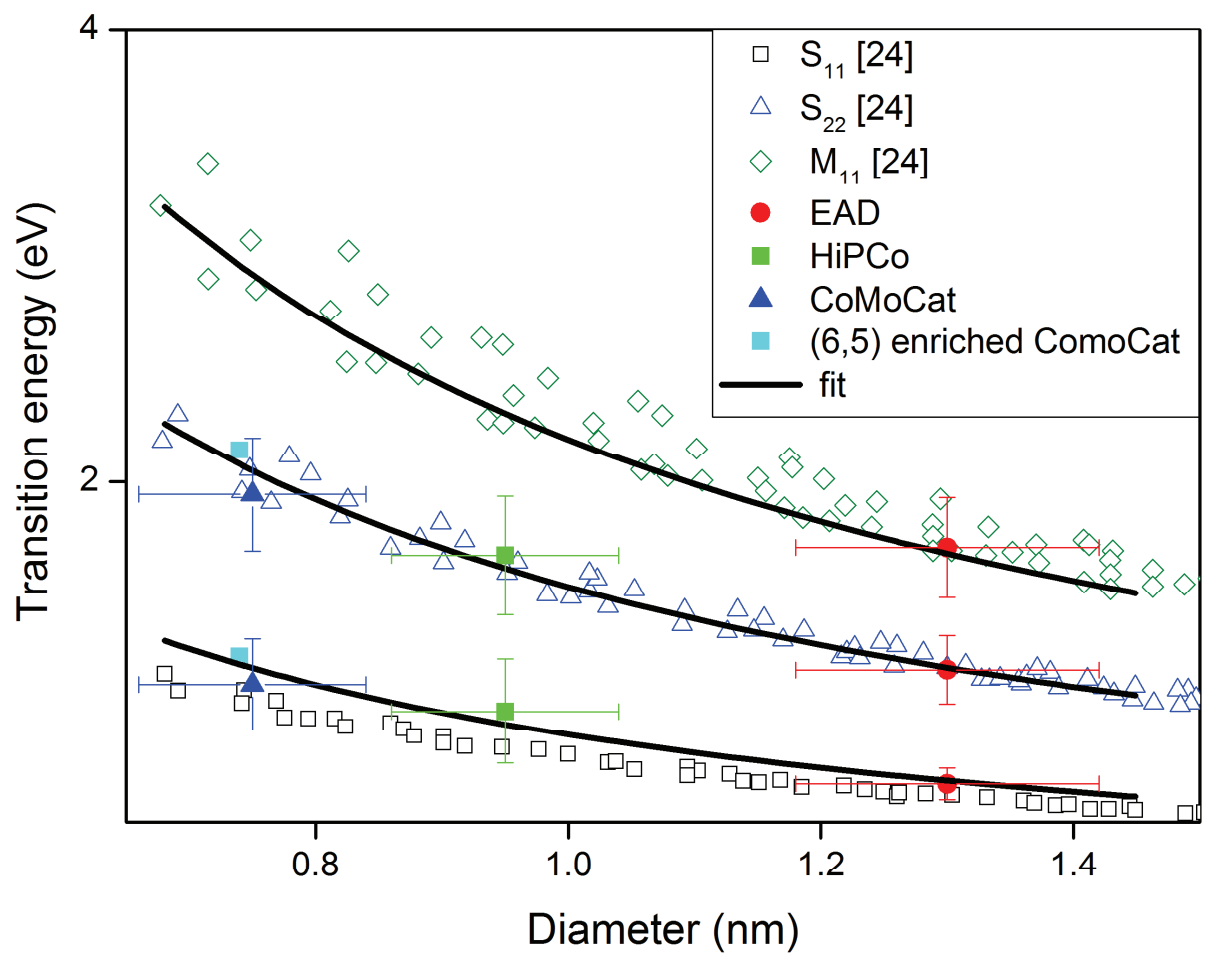

Fig. 3- Comparison between the Kataura plot of experimental data (filled symbol), their fitting from equation (2-3) (black line) and the calculated values from the tight binding approximation[24] (empty symbol).

\subsection{Electronic properties of SWCNTs:}

In the $0.07 \mathrm{eV}-0.40 \mathrm{eV}$ range, the real part of the dielectric function become more and more negative while the imaginary parts abruptly increases as the photon energy decreases. This metallic behaviour was previously observed[26] and involves the free charge carrier motion in the SWCNT network. Note that SC-SWCNTs are known to be p-type in air[15]. Several works have previously reported the presence of an absorption band at $17 \mathrm{meV}$ [18,26-28]. This band was attributed to the bandgap of M-SWCNTs induced by curvature effect. However, due to our limited spectral range, this band cannot be observed in Figure 2. As shown in figure 2, 
the SWCNT dielectric function in the infrared spectral range can be modelled by a Drude dispersion law[10, 26]:

$\varepsilon=\varepsilon_{\infty}-\frac{\omega_{p}^{2}}{\omega(\omega+i \gamma)}$,

where $\varepsilon$ and $\omega$ are the effective dielectric function of the SWCNT film and the photon energy, respectively. Three parameters are adjusted $\varepsilon_{\infty}, \omega_{\mathrm{p}}$ et $\gamma$ which correspond to the dielectric function at high energy, the plasma energy and the damping constant of free charge carrier. To give more insights on the electronic properties of SWCNT films, the free charge carrier concentration $\mathrm{n}$ and the conductivity $\sigma$ of SWCNT film calculated from equations (5) and (6) are represented in Fig.4a.

$\sigma=\frac{\varepsilon_{0} \varepsilon_{\infty} \omega_{p}^{2}}{\gamma}$, (5) $n=\frac{m \varepsilon_{0} \omega_{p}^{2}}{e^{2}}$

e and $\varepsilon_{0}$ are the elementary charge and vacuum permittivity. The effective mass $m$ of charge carriers in carbon nanotubes is $0.9 * 10^{-31} \mathrm{~kg}$ [29]. The conductivity obtained from ellipsometry is compared to DC conductivity estimated from Van Der Paw electrical measurements (Figure 4a). Despite a limited ellipsometric spectral range, the film conductivity values obtain from ellipsometric measurements deviates by less than $6 \%$ from the estimated ones from Van Der Pauw electrical measurements confirming correctness of the ellipsometric data analysis. In other words, the additional low frequency band reported in references[18,26-28] at $17 \mathrm{meV}$ has a negligible effect on the DC conductivity. By considering that SWCNTs are p-doped in air[15], the free charge carrier concentration can be assimilated to the hole concentration. The hole concentration and the film conductivity increase with the SWCNT diameter (Fig.4a). The charge carrier density of SWCNTs is close to the charge carrier density of graphite [30]. Despite CoMoCat and $(6,5)$ SWCNTs have similar diameter, their charge carrier concentration and conductivity differ. Indeed, the number of M-SWCNTs and so the concentration in free charge carriers is smaller in $(6,5)$ SWCNTs. By considering the 
approach introduced by Marulada et al.[29,31], the hole carrier concentration of SWCNTs is defined by:

$$
n=\frac{1}{0.25 \pi\left((d+h)^{2}-d^{2}\right)} \int_{\infty}^{E_{f}} D(E)(1-f(E)) d E,(7)
$$

where $\mathrm{f}(\mathrm{E})$ is the Fermi Dirac distribution, $\mathrm{h}$ the effective wall thickness of SWCNTs equals to $0.617 \AA$ and $d$ the SWCNT diameter. The DOS (D(E)) of SWCNTs is given by[27,31]:

$$
D(E)=\frac{8}{3 \pi \gamma_{0} a_{c c}} \sum_{i}^{\text {all bands }} g_{i}(E),(8) \text { with } g_{i}(E)=\left\{\begin{array}{c}
\frac{|E|}{\sqrt{E^{2}-E_{c, i}^{2}}}-s i|E|>E_{c, i} \\
1 \text { si } E_{c, i}=0 \\
0 \text { si }|E|<E_{c, i}
\end{array}\right.
$$

$E_{c, i}$ is the minimum energy value of the $i^{\text {th }}$ conduction band. By considering a rigid band model whereby doping shifts the Fermi level without affecting the band structure, this approach can also be used to compute the charge carrier density of doped SWCNTs by air. Thus, simulations have been made for undoped and p-doped carbon nanotubes by assuming the Fermi level in the mid-gap of SWCNTs and shifted by $\mathrm{E}_{\mathrm{g}(6,5)} / 2 \sim 0.3 \mathrm{eV}$ toward the valence band[32-33], respectively. Although no accurate value of the Fermi level shift has been found in the literature, simulations performed in Figure $4 \mathrm{~b}$ give qualitative information used to highlight the electronic properties of SWCNTs. 


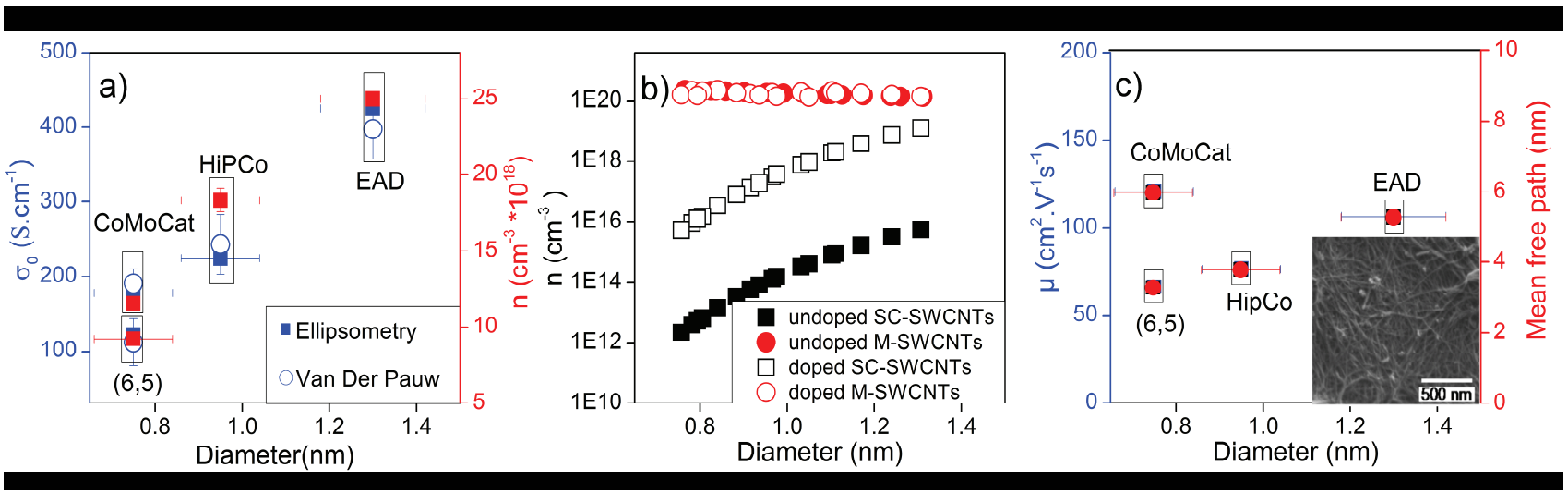

Fig. 4- (a) Variation of the DC conductivity and charge carrier density measured by ellipsometry, with the SWCNT diameter (filled symbol). The DC conductivity extracted from Van Der Pauw measurements is also reported (empty symbol). (b) Theoretical charge carrier density of doped $\left(\left|\Delta \mathrm{E}_{\mathrm{f}}\right|=0.3 \mathrm{eV}\right.$, empty symbol) and undoped $\left(\left|\Delta \mathrm{E}_{\mathrm{f}}\right|=\mathbf{0} \mathrm{eV}\right.$, filled symbol) M-SWCNTs and SC-SWCNT. (c) Charge carrier mobility and mean free path versus the SWCNT diameter. In inset, a typical SEM image of the SWCNT surface.

In accordance with Marulada et al. [29,31], simulations presented in the Figure $4 \mathrm{~b}$ show that the charge carrier concentration increases by three orders of magnitude for SC-SWCNTs while it slightly decreases for M-SWCNTs as the diameter increases. Due to the higher DOS of M-SWCNTs close to the Fermi energy, the charge carrier concentration in SC-SWCNTs is smaller than the metallic one. Since M-SCWNT has constant DOS near the Fermi level, the Fermi level shift induced by doping has little effect on the charge carrier concentration. On the contrary, doped SC-SWCNTs have a higher charge carrier concentration than undoped SC-SWCNTs. Thus, air doping may induce a drastic increase of the concentration of the SCSWCNT hole charge carriers. The charge carrier concentration estimated from ellipsometry is smaller than the calculated charge carrier concentration of M-SWCNTs but slightly higher than the doped SC-SWCNT one. Indeed, the films are composed of a mixture of both kinds of SWCNTs. As the optical $\mathrm{M}_{11}$ band of M-SWCNTs is more and more pronounced as the 
SWCNT mean diameter increases, we can conclude that the variations of the measured charge carrier concentration and so the DC conductivity can be attributed to the increase of both, the charge carrier concentration in doped SC-SWCNTs and the M-SWCNT concentration.

By considering that all holes which participate to the conduction have an energy close to the Fermi energy, the hole carrier mobility $\mu$ and mean free path $1_{\mathrm{f}}$ are both related to the damping constant:

$\mu=\frac{e}{\gamma m}(10)$ and $l_{f}=\frac{v_{f}}{\gamma}(11)$

where $v_{f}=8.8^{*} 10^{5} \mathrm{~m} \cdot \mathrm{s}^{-1}$ is the Fermi velocity[29]. Figure $4 \mathrm{c}$ shows that the variations of hole mobility $\mu$ and mean free path as a function of nanotubes diameter are similar. Large dispersion of mobility around the $75 \mathrm{~cm}^{2} \mathrm{~V}^{-1} \mathrm{~s}^{-1}$ value is observed. The mobility is smaller than the measured one on individual nanotubes[34]. Despite a micrometer length ballistic transport was reported for individual SWCNT[35], the mean free path of charge carrier is in the nanometer scale. The hole mobility and mean free path are both related to the scattering events and potential barriers that occur in the conduction channel. As shown by scanning electron microscopy (inset of figure 4c), the films are composed of highly entangled SWCNTs. Schottky barrier between M-SWCNTs and SC-SWCNTs drastically reduces the mean free path of carriers. In addition, potential barriers are also present at the junction of two M-SWCNTs or two SC-SWCNTs. In other words, the small mean free path and mobility values come from the large number of SWCNT-SWCNT contacts in the film. 


\subsection{Diameter dependence of the $\pi$-plasmon band}

The broad band observed in the $3.95-4.50 \mathrm{eV}$ range (Fig.2) is attributed to the $\pi$-plasmon resonance induced by the collective oscillation of $\pi$-electrons polarised along the tube axis which arises from the electronic transitions near the M-point of the Brillouin zone. In contrast with transitions between VHS, the position of the $\pi$-plasmon band is blueshifted from $3.95 \mathrm{eV}$ to $4.50 \mathrm{eV}$ as the nanotubes diameter increase. In accordance with our results, a blueshift of the $\pi$-plasmon band as the SWCNT diameter increases, was reported in recent ab-initio calculations which take into account local field effects[36]. As reported by Park et al.[13], the $\pi$-plasmon band energy depends on the concentration ratio between M-SWCNTs and SCSWCNTs i.e. the charge carrier concentration. Moreover, a large blue shift of the $\pi$-plasmon band accompanied by an increase of SWCNT film reflectivity was reported for hydrogenated or fluorinated SWCNT[37]. Hydrogenation and fluorination are known to dope SWCNTs. In other words, the variation of $\pi$-plasmon band energy depends on the number of transferred charges. By assuming that all free charge carriers contribute to the collective surface oscillation of the $\pi$ electrons, a quadratic relation, derived from free electron gas model[38], can be found after some calculations between the $\pi$-plasmon energy $\left(\omega_{\pi}\right)$ and the charge carrier concentration:

$\omega_{\pi}^{2}=\omega_{0}^{2}+\frac{e^{2}}{2 \varepsilon_{0} m} n$,

The first term of equation (12) is associated to the $\pi-\pi^{*}$ interband oscillator energy $\omega_{0}$, while the second term represents the surface plasmon energy of $\pi$ electrons. Note that due to the expansion of the wave function of $\pi$-electrons over the SWCNT diameter, SWCNTs are considered, in first approximation, as a solid cylinder[38]. In agreement with equation (12), a linear behaviour is obtained between $\omega_{\pi}^{2}$ and $\mathrm{n}$ (Fig.5), confirming the correlation between 
free charge carriers and the $\pi$-plasmon band energy. Note that the slope corresponds to the calculated one from equation (12). The $\pi-\pi^{*}$ interband oscillator energy is estimated at 3.7 $\pm 0.1 \mathrm{eV}$. Thus, we can conclude that the blue shift of the $\pi$-plasmon band, observed by increasing the SWCNT diameter, is attributed to the increase of the free charge carrier concentration.

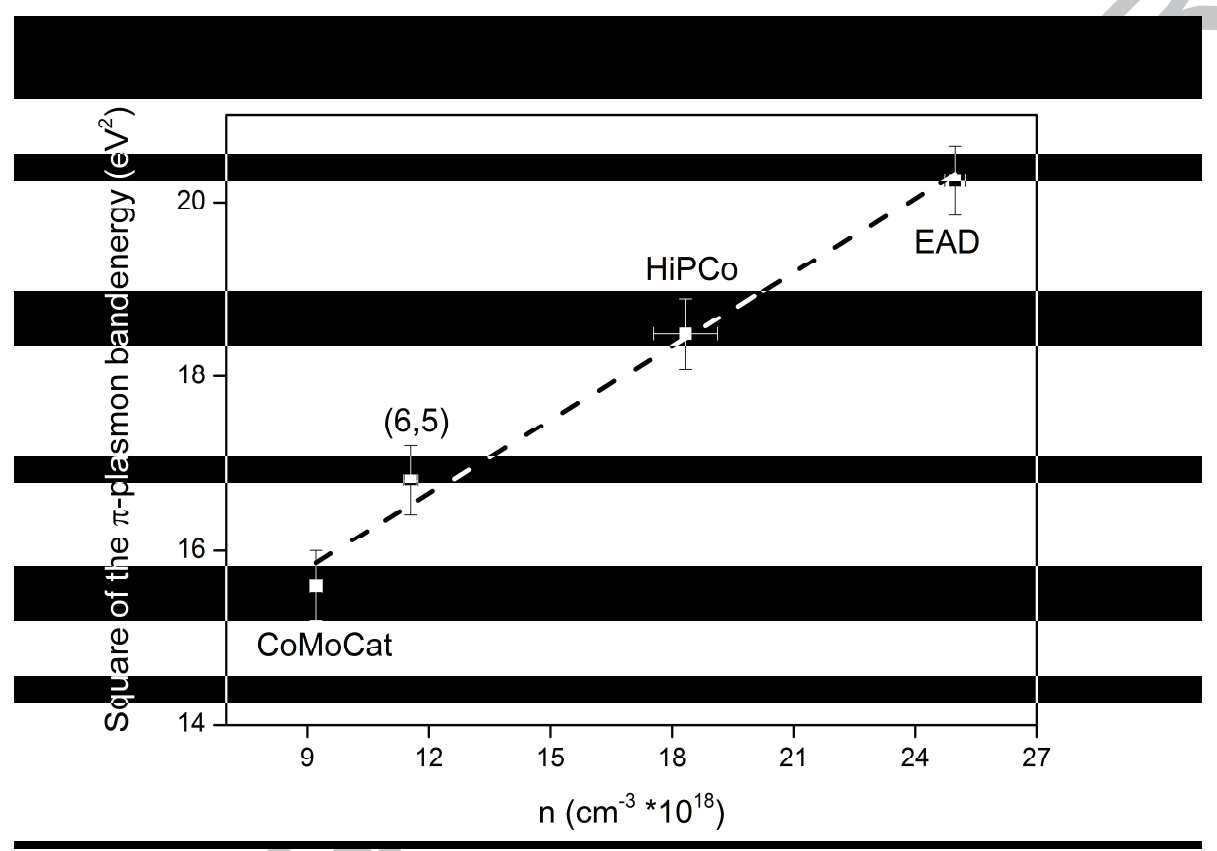

Fig. 5- Square of the $\pi$-plasmon energy versus the charge carrier density.

\section{Conclusion}

In summary, we have highlighted the dependences between the diameter distribution and the complex dielectric functions of SWCNTs. Due to the strong one dimensional confinement, the energy of optical interband transition between VHS is redshifted when the diameter increases. The DC electronic properties of SWCNT are evaluated by analysing the infrared part of the dielectric function by a Drude model. The mean free path of charge carrier is drastically reduced by the large number of SWCNT contact. Finally, we have demonstrated that the position of the $\pi$-plasmon band is correlated to the concentration of charge carrier. 


\section{References:}

[1] Fanchini G., Miller S., Parekh B. B., Chhowalla M. Optical anisotropy in single-walled carbon nanotube thin films: implications for transparent and conducting electrodes in organic photovoltaics. Nano Lett. 2008; 8(8):2176-9.

[2] Nong H., Gicquel-Guezo M., Bramerie L., Perrin M., Grillot F., Levallois, C., Maalouf, A. Loualiche, S. A direct comparison of single-walled carbon nanotubes and quantum-wells based subpicosecond saturable absorbers for all optical signal regeneration at $1.55 \mu \mathrm{m}$. Appl. Phys. Lett. 2010;96(6):061109-061109.

[3] Della Valle G., Osellame R., Galzerano G., Chiodo N., Cerullo G., Laporta P., Svelto O., Morgner U., Rozhin A. G., Scardaci V., Ferrari A. C. Passive mode locking by carbon nanotubes in a femtosecond laser written waveguide laser. Appl. Phys. Lett. 2006;89(23):231115-231115.

[4] Maine S., Koechlin C., Fleurier R., Haidar R., Bardou N., Dupuis C., Attal-Trétout B., Mérel P., Deschamps J., Loiseau A., Pelouard, J.-L. Mid - infrared detectors based on carbon nanotube films. Phys. Status Solidi C 2010;7(11-12):2743-2746.

[5] Heinze S., Tersoff J., Martel R., Derycke V., Appenzeller J., Avouris P. Carbon Nanotubes as Schottky Barrier Transistors. Phys. Rev. Lett. 2002;89(10):106801-106801. 
[6] Battie Y., Gorintin L., Ducloux O., Thobois P., Bondavalli P., Feugnet G., Loiseau A. Thickness dependent sensing mechanism in sorted semi-conducting single walled nanotube based sensors. Analyst 2012;137(9):2151-2157.

[7] Guo G. Y., Chu K. C. Wang, D.-S., Duan, C.-G. Linear and nonlinear optical properties of carbon nanotubes from first-principles calculations. Phys. Rev. B 2004;69(20):205416205416.

[8] Lin, M. F. Optical spectra of single-wall carbon nanotube bundles. Phys. Rev. B 2000;62(19):13153-9.

[9] Battie Y., Jamon D., En Naciri A., Lauret J. S., Loiseau A. Chirality distribution in single walled carbon nanotube films by spectroscopic ellipsometry. Appl. Phys. Lett. 2013;102(9):091909-091909.

[10] Fanchini G., Emrah Unalan H., Chhowalla M. Optoelectronic properties of transparent and conducting single-wall carbon nanotube thin films. Appl. Phys. Lett. 2006;88(19):191919-191919.

[11] Battie Y., Jamon D., Lauret J. S., En Naciri A., Broch L., Loiseau A. Optical anisotropy of single walled carbon nanotubes investigated by spectroscopic ellipsometry. Carbon 2012;50(12):4673-4679. 
[12] Fanchini G., Emrah Unalan, H., Chhowalla M. Modification of transparent and conducting single wall carbon nanotube thin films via bromine functionalization. Appl. Phys. Lett. 2007;90(9):092114-092114.

[13] Park Y. R., Kim W. J., Ko M. J., Min N. K., Lee C. J. Investigation of ultraviolet optical properties of semiconducting-enriched and metal-enriched single-walled carbon nanotube networks using spectroscopic ellipsometry. Nanoscale 2012;4(20):6532-36.

[14] Green A. A., Hersam M. C. Nearly Single - Chirality Single - Walled Carbon Nanotubes Produced via Orthogonal Iterative Density Gradient Ultracentrifugation. Adv. Mater. 2011;23(19):2185-2190.

[15] Posseckardt J., Battie Y., Fleurier R., Lauret J. S., Loiseau A., Jost O., Mertig M. Improved sorting of carbon nanotubes according to electronic type by density gradient ultracentrifugation. Phys. Status Solidi B 2010;247(11-12):2687-90.

[16] Azzam R. M. A., Bashara N. M. Ellipsometry and polarized light. North-Holland Personal Library, 1987.

[17] Bachilo S. M., Strano M. S., Kittrell C., Hauge R. H., Smalley R. E., Weisman R. B. Structure-assigned optical spectra of single-walled carbon nanotubes. Science 2002;298(5602):2361-2366.

[18] Pekker A., Kamarás K. Wide-range optical studies on various single-walled carbon nanotubes: Origin of the low-energy gap. Phys. Rev. B 2011;84(7):075475-075475. 
[19] Green A. A., Hersam M. C. Colored semitransparent conductive coating consisting of manodisperse metallic single walled carbon nanotubes. Nano Letters 2008; 8(5):1417-1422.

[20] Liu H., Tanaka T., Kataura H. One-step separation of high-purity $(6,5)$ carbon nanotubes by multicolumn gel chromatography. Physica Status Solidi B 2011;248(11):2524-2527.

[21] Knupfer M., Pichler T., Golden M.S., Fink J., Rinzler A., Smalley R.E. Electron energyloss spectroscopy studies of single wall carbon nanotubes.

[22] Lauret J-S., Voisin C., Cassabois G., Delalande C., Roussignol Ph., Jost O., Capes L. Ultrafast carrier dynamics in single wall carbon nanotubes. Physical review letters 2003;90(5):057404.

[23] Lin H., Lagoute J., Repain V., Chacon C., Girard Y., Lauret J.-S., Ducastelle F., Loiseau A., Rousset S. Many-body effects in electronic bandgaps of carbon nanotubes measured by scanning tunnelling spectroscopy. Nature materials 2010;9(3):235-8.

[24] Kataura H., Kumazawa Y., Maniwa Y., Umezu I., Suzuki S., Ohtsukac Y., Achiba Y. Optical properties of single-wall carbon nanotubes. Synth. Met. 1999;103(1):2555-8.

[25] Samsonidze G. G., Saito R., Jorio A., Pimenta M. A., Filho A. G. S., Grüneis F A., Dresselhaus D G., Dresselhausa M. S. The concept of cutting lines in carbon nanotube science. J. Nanosci. Nanotech. 2003;3(6):431-58. 
[26] Maine S., Koechlin C., Rennesson S., Jaeck J., Salort S., Chassagne B., Pardo F., Pelouard J.L., Haïdar R. Complex optical index of single wall carbon nanotube films from the near-infrared to the terahertz spectral range. Appl. Opt. 2012;51(15):3031-35.

[27] Kamaras K., Pekker A., Bruckner M., Borondics F., Rinzler A. G., Tanner D. B., Itkis M. E., Haddon R. C., Tan Y., Resasco D. E. Wide - range optical spectra of carbon nanotubes: a comparative study. Phys. Stat. Sol. B 2008;245(10):2229-32.

[28] Ugawa A., Rinzler A. G., Tanner D. B. Far-infrared gaps in single-wall carbon nanotubes. Phys. Rev. B 1999;60(16):305-8.

[29] J. M. Marulanda, Current transport modeling of carbon nanotubes: Concepts, analysis, and design. VDM Verlag, 2009.

[30] Dillon R.O., Spain I. L., McClure J.W. Electronic energy band parameters of graphite and their dependence on pressure, temperature and acceptor concentration. J. Phys. Chem. Solids 1977;38(6):635-45.

[31] M.Marulanda J., Srivastava A. Carrier density and effective mass calculations in carbon nanotubes. Phys. Status Solidi B 2008;245(11):2558-62.

[32] Blackburn J. L., Barnes T. M., Beard M. C., Kim Y.-H., Tenent R. C., McDonald T.J., To B., Coutts T.J., Heben M.J. Transparent conductive single-walled carbon nanotube networks with precisely tunable ratios of semiconducting and metallic nanotubes. ACS Nano 2008;2(6):1266-74. 
[33] Tchernatinsky A., Desai S., Sumanasekera G. U., Jayanthi C. S., Wu S. Y., Nagabhirava B., Alphenaar B. Adsorption of oxygen molecules on individual single-wall carbon nanotubes. J. of Appl. Phys. 2006;99(3):034306-034306.

[34] Dürkop T., Getty S. A., Cobas E., Fuhrer M. S. Extraordinary mobility in semiconducting carbon nanotubes. Nano Lett. 2004;4(1):35-9.

[35] Bachtold A., Fuhrer M. S., Plyasunov S., Forero M., Anderson E. H., Zettl A., McEuen P.L. Scanned probe microscopy of electronic transport in carbon nanotubes. Phys. Rev. Lett. 2000;84(26):6082-5.

[36] Marinopoulos A. G., Reining L., Rubio, A. Ab initio study of the dielectric response of crystalline ropes of metallic single-walled carbon nanotubes: Tube-diameter and helicity effects. Phys. Rev. B 2008;78(23):235428-235428.

[37] Lee K. J., Lee H., Lee J. Y., Lim S. C., An K. H., Lee Y.H. Optical properties of hydrogen-and fluorine-doped single-walled carbon nanotubes. J. Kor. Phys. Soc. 2005;46(4):906-12.

[38] Pitarke J. M., Garcia-Vidal F. J. Electronic response of aligned multishell carbon nanotubes. Phys. Rev. B 2001;63(7):073404-073404. 